\title{
ON PROJECTIVE MODULES OF FINITE RANK
}

\author{
WOLMER V. VASCONCELOS ${ }^{1}$
}

One of the aims of this paper is to answer the following question: Let $R$ be a commutative ring for which projective ideals are finitely generated; is the same valid in $R[x]$, the polynomial ring in one variable over $R$ ? A Hilbert basis type of argument does not seem to lead directly to a solution. Instead we were taken to consider a special case of the following problem: Let $\left(X, O_{X}\right)$ be a prescheme and $M$ a quasi-coherent $O_{X}$-module with finitely generated stalks; when is $M$ of finite type? Examples abound where this is not so and here it is shown that a ring for which a projective module with finitely generated localizations is always finitely generated, is precisely one of the kind mentioned above (Theorem 2.1). Such a ring $R$ could also be characterized as "any finitely generated flat module is projective."

1. Preliminaries. Throughout $R$ will denote a commutative ring. Let $M$ be a projective $R$-module; for each prime $p, M_{p}$, the localization of $M$ with respect to $R-p$, is by [4] a free $R_{p}$-module, with a basis of cardinality $\rho_{M}(\boldsymbol{p}) \cdot \rho_{M}$ is the so-called rank function of $M$ and here it will be assumed that $\rho_{M}$ takes only finite values.

The trace of $M$ is defined to be the image of the map $M \otimes_{R} \operatorname{Hom}_{R}(M, R) \rightarrow R, m \otimes f \rightarrow f(m)$; it is denoted by $\tau_{R}(M)$. If $M \oplus N=F$ (free), it is clear that $\tau_{R}(M)$ is the ideal of $R$ generated by the coordinates of all elements in $M$, for any basis chosen in $F$. It follows that for any homorphism $R \rightarrow S, \tau_{S}\left(M \otimes_{R} S\right)=\tau_{R}(M) S$. In particular, if $\boldsymbol{p}$ is a prime ideal, $\tau_{R}(M)_{p}=\tau_{R_{p}}\left(M_{p}\right)=(0)$ or (1) depending on whether $M_{p}=(0)$ or $M_{p} \supseteq(0)$. Thus $\left(\tau_{R}(M)\right)^{2}=\tau_{R}(M)$ and $\tau_{R}(M)$ is generated by an idempotent if it is finitely generated; also, $\tau_{R}(M) M=M$. For later reference, we isolate part of this discussion into

(1.1) Lemma. The support of a projective module $M$ is open in $\operatorname{Spec} R$.

Proof. In fact, it was shown that Supp $M=\{$ all primes not containing $\left.\tau_{R}(M)\right\}$. (See also [5].)

In its simplest case the trace and the finite generation of a projective module are related by

(1.2) Lemma. Let $M$ be a projective module such that for each prime

Received by the editors November 15, 1968.

1 The author gratefully acknowledges partial support from the National Science Foundation under grant GP-8619. 
$p, M_{p} \cong R_{p}$ or $(0)$. Then $M$ is finitely generated iff $\tau_{R}(M)$ is finitely generated.

Proof. If $\tau_{R}(M)$ is finitely generated, as remarked earlier, it can be generated by an idempotent $\tau_{R}(M)=R e$ and $M$ can be viewed as a projective module over $R e$ with unit trace. Changing notation can write

$$
\sum_{i=1}^{n} f_{i}\left(m_{i}\right)=1
$$

with $f_{i} \in \operatorname{Hom}_{R}(M, R)$. Now, for each maximal ideal $\boldsymbol{p}, M / \mathbf{p} M \cong R / \boldsymbol{p}$ and an element of $M$ generates $M_{p}$ provided it is in $M-p M$. As $\left(^{*}\right)$ makes it impossible for all $x_{i}$ 's to be in $p M$ it is clear that $x_{1}, \cdots, x_{n}$ generate $M$ for they do so locally. The converse is trivial.

This can be extended to higher ranks through the device of exterior products. If $M$ is a projective module its exterior powers are also projective as $M \oplus N=F$ (free) implies

$$
\stackrel{r}{\Lambda}(M \oplus N)=\Lambda^{r} M \oplus \cdots \oplus \Lambda^{r} N=\Lambda^{r} F
$$

and the exterior powers of a free module are clearly free [3].

(1.3) Proposition. Let $M$ be a projective module of constant finite rank (i.e. $\rho_{M}(\boldsymbol{p})=r$ for any prime $\boldsymbol{p}, r$ a fixed integer); then $M$ is finitely generated.

Proof. If $r$ is the integer above, then $\wedge^{r} M$ is a projective module of rank 1 and by (1.2) finitely generated. Pick $m_{1}, \cdots, m_{n}$ elements in $M$ such that their $r$-exterior products generate $\Lambda^{r} M$. To check that those elements generate $M$ it is enough to do it locally when it becomes clear.

Finally, if the rank function is no longer constant, one has

(1.4) Proposition. Let $M$ be a projective module with $M_{p}$ finitely generated over $R_{p}$ and bounded rank. Then $M$ is finitely generated iff $\rho_{M}$ is locally constant.

Proof. The limitation on the ranks of the localizations of $M$ can be expressed by saying that there is a last integer $r$ for which $\Lambda^{r} M \neq(0)$. Let $I_{r}$ be the trace of $\Lambda^{r} M$. As $\rho_{M}$ is locally constant, supp $\Lambda^{r} M$ is then both open and closed. There is then an ideal $J_{r}$ with $I_{r}+J_{r}=R, I_{r} \cap J_{r}=$ nilideal. Through standard techniques, an idempotent $e$ can be found in $I_{r}$ such that $(e)+J_{r}=R$. As $I_{r}$ is locally $(0)$ or (1) it is easy to verify that $I_{r}=(e)$. Decompose $R$ into 
$R e \oplus R(1-e)$ and, similarly, $M=e M \oplus(1-e) M$. As an $R e$-module, $e M$ is projective of constant rank $r$. As for $(1-e) M$, it has rank $<r$ as a projective $R(1-e)$-module and inherits the rank function of $M$ on $\operatorname{Spec}(R(1-e))$. (1.3) and induction finish the proof.

2. Main results. The question raised at the outset is now answered. It will follow from

(2.1) THEOREM. Let $R$ be a commutative ring. The following are equivalent for $R$-modules:

(1) Finitely generated flat modules are projective.

(2) A locally finitely generated projective module is finitely generated.

(3) Projective ideals are finitely generated.

Proof. (1) $\Rightarrow(2)$ : Let $M$ be a projective module. If $I_{r}$ denotes the trace of $\Lambda^{r} M$, then $R / I_{r}$ is a flat module and thus projective, and $I_{r}$ is generated by an idempotent. If $\rho_{M}$ is bounded we are done by (1.4); if not, we get a decreasing sequence $I_{1} \supseteq I_{2} \supseteq \cdots$ of ideals generated by idempotents, say $I_{i}=\left(e_{i}\right)$. Consider the sequence

$$
\left(f_{1}\right) \subseteq\left(f_{2}\right) \subseteq \cdots, \quad f_{i}=1-e_{i}
$$

Let $I=\mathrm{U}\left(f_{i}\right) . I \neq R$ as no $e_{i}=0$. But $I$ is locally (0) or (1) and, as before, going over to $R / I$, we conclude that $I$ is finitely generated, i.e., $I=\left(f_{n}\right)$ for some $n$. But for any prime $\boldsymbol{p} \supseteq\left(f_{n}\right)$ we get that $\rho_{M}(\boldsymbol{p})$ is nonfinite.

$(2) \Rightarrow(3)$ : Obvious.

$(3) \Rightarrow(1)$ : For this we use an argument inspired by [1]. It is enough to show that cyclic flat modules are projective [6], [7]. Let $R / I$ be one such, $I \neq 0$. Then $I$ is locally (0) or (1). Let $a_{1}$ be a nonzero element of $I$; then $\left(a_{1}\right)=a_{1} I$, checked by localization. Write $a_{1}=a_{1} a_{2}$, $a_{2} \in I$. Proceeding in this fashion we get a sequence of principal ideals $\left(a_{1}\right) \subseteq\left(a_{2}\right) \subseteq \cdots$ with $a_{i}=a_{i} a_{i+1}$. If it becomes stationary at $\left(a_{n}\right)$, say, $a_{n+1}=\lambda a_{n}$ and $a_{n+1}^{2}=a_{n+1} \lambda a_{n}=\lambda a_{n}=a_{n+1}$. If $\left(a_{n}\right) \neq I$, we split $I=\left(a_{n}\right) \oplus I^{\prime}$, with $I^{\prime}$ also being locally (0) or (1). Doing the same for $I^{\prime}$ we get a similar sequence $\left(a_{1}^{\prime}\right) \subseteq\left(a_{2}^{\prime}\right) \subseteq \cdots$, and thus a longer sequence

$$
\left(a_{1}\right) \subseteq\left(a_{2}\right) \subseteq \cdots \subseteq\left(a_{n}\right) \subseteq\left(a_{n}+a_{1}^{\prime}\right) \subseteq\left(a_{n}+a_{2}^{\prime}\right) \subseteq \cdots
$$

In this way either $I$ turns out to be generated by an idempotent (as wanted) or we get a strictly increasing sequence $\left(x_{1}\right) \subseteq\left(x_{2}\right) \subseteq \ldots$. with $x_{i}=x_{i} x_{i+1}$. Assume this is the case; write $J=U\left(x_{i}\right)$. By exhibiting a "dual basis" for $J[2$, p. 132] we prove $J$ to be projective. Let $f_{i} \in \operatorname{Hom}_{R}(J, R)$ be defined: $f_{1}(x)=x ; f_{i}(x)=\left(1-x_{i-1}\right) x, i>1$. We 
claim that $f_{i}(x)=0$ for all large $i$ 's. If, say, $x=\lambda x_{n-2}, f_{n}(x)$ $=\left(1-a_{n-1}\right) \lambda a_{n-2}=0$. Also, $\sum f_{i}(x) x_{i}=x$. This contradicts (3) and $I$ is finitely generated.

We can now state

(2.2) COROLlARY. Let $R$ be a ring for which projective ideals are finitely generated. Then the polynomial ring, $R[x]$, enjoys the same property.

Proof. It is enough to show that the support of a projective module is closed. For a projective $R[x]$-module $M$ one checks easily that Supp $M$ is defined by the ideal $\tau_{R}(M / x M) \cdot R[x]$. As $\tau_{R}(M / x M)$ is generated by an idempotent, we are through.

3. Remarks. It is not always the case that the rank of a locally finitely generated projective is bounded. Consider $R$ to be a regular (von Neumann) ring with countably many prime ideals (e.g. $R=$ subring of $\prod^{\infty} k, k$ a field, generated by $k e, e=$ identity and the basic idempotents). Number the prime ideals: $\boldsymbol{m}_{0}, \boldsymbol{m}_{\mathbf{1}}, \cdots$ Each is projective. Let

$$
M=\boldsymbol{m}_{0} \oplus \boldsymbol{m}_{0} \cdot \boldsymbol{m}_{1} \oplus \cdots \oplus \boldsymbol{m}_{0} \cdot \boldsymbol{m}_{1} \cdots \boldsymbol{m}_{n} \oplus \cdots ;
$$

it is projective and $M_{m_{n}} \cong\left(R_{m_{n}}\right)^{n}$.

What are the rings of (2.1)? Obviously domains, semilocal rings, polynomial or power series over them. If $R$ is semihereditary, it must be a direct sum of Prufer domains [7]. It seems plausible that if $R$ satisfies the conditions of (2.1), the same holds in $R\left[x_{\alpha}\right.$ 's $]=$ polynomial ring in any number of variables.

\section{BiBLIOGRAPHY}

1. H. Bass, Finitistic dimension and a homological generalization of semi-primary rings, Trans. Amer. Math. Soc. 95 (1960), 466-492.

2. H. Cartan and S. Eilenberg, Homological algebra, Princeton Univ. Press, Princeton, N. J., 1956. 1956.

3. C. Chevalley, Fundamental concepts of algebra, Academic Press, New York,

4. I. Kaplansky, Projective modules, Ann. of Math. 68 (1958), 372-377.

5. D. Lazard, Disconnexités des spectres d'anneaux et des préschémas, Bull. Soc. Math. France 95 (1967), 95-108.

6. K. Mount, Some remarks on Fitting's invariants, Pacific J. Math. 13 (1963), 1453-1357.

7. W. V. Vasconcelos, On finitely generated flat modules, Trans. Amer. Math. Soc. 138 (1969), 505-512.

RUTGERS UNIVERSITY 